\title{
Modeling and Simulation of Hydraulic Mount Dynamic
}

\author{
Y.Q.WANG \& B. QIAN \\ Anhui Technical College of Mechanical and Electrical Engineering, Wuhu 241006, China
}

S.S PENG

School of Mechanical and Automotive Engineering, Anhui Polytechnic University, Wuhu 241000, China

\begin{abstract}
This paper basing on the linear model used the switched system theory and started with the actual physical work process of the decoupling disc hydraulic suspension to divide the vibration motion state into two kinds of work modes, four kinds of basic working process, make switched strategy pattern between the work modes and establish the switched system model of the decoupling disc hydraulic mount. Finally, the validity of the model was verified by comparing the decoupling disc hydraulic mount dynamic characteristics test with the simulation results of the model.
\end{abstract}

KEYWORD: Hydraulic mount; Dynamic characteristic; Simulation

\section{INTRODUCTION}

The hydraulic mount is a vibration- isolating device that was parallel connection by the rubber bearing and the hydraulic system[1]. The structure of the hydraulic suspension has multiple types, but its basic principle is the same. The decoupling hydraulic disc suspension is widely used because of its relatively simple structure, low manufacturing cost, and the properties of its vibration isolation can well meet the requirements of automotive power vibration. The dynamic characteristic of decoupling disc hydraulic mount, however, is very complicated, so it is important to accurately establish its mathematical model[2].

\section{MECHANICS MODEL OF THE HYDRAULIC MOUNT}

Experiments show that the fluid in the hydraulic mount has only large influence of the suspension in vertical dynamic characteristics, little influence of its lateral dynamic characteristics, so the paper only establish the vertical mechanics model of the hydraulic mount shown in figure 1 . In the figure, $X_{r}(t)$ is the displacement excitation. $F_{T}(t)$ is the exciting force of the hydraulic suspension base. $C_{u}$ and $C_{d}$ are the volume compliance of the liquid chamber and a lower liquid chamber, the reciprocal of which is $K_{u}=1 / C_{u}, K_{d}=1 / C_{d}$ are the volume stiffness of the upper liquid chamber and the lower liquid chamber. $K_{r}$ and $B_{r}$ are respectively the main rubber spring stiffness and the damping coefficient. $I_{i}$ is the inertial coefficient of the liquid mass in the inertia passage. $R_{i}$ is the damping coefficient when the liquid flowed in the inertia channel. $I_{d}$ is the inertial coefficient of the liquid mass in a decoupling channel. $R_{d}$ is the damping coefficient when the liquid flowed in the decoupling channel. Let $A_{p}$ is the equivalent piston area of the rubber spring. $Q_{i}(t)$ and $Q_{d}(t)$ are respectively the liquid volume that flowed through the inertia channel and the decoupling channel. $P_{1}(t)$ and $P_{2}(t)$ are respectively the pressure of the upper liquid chamber and the lower liquid chamber.

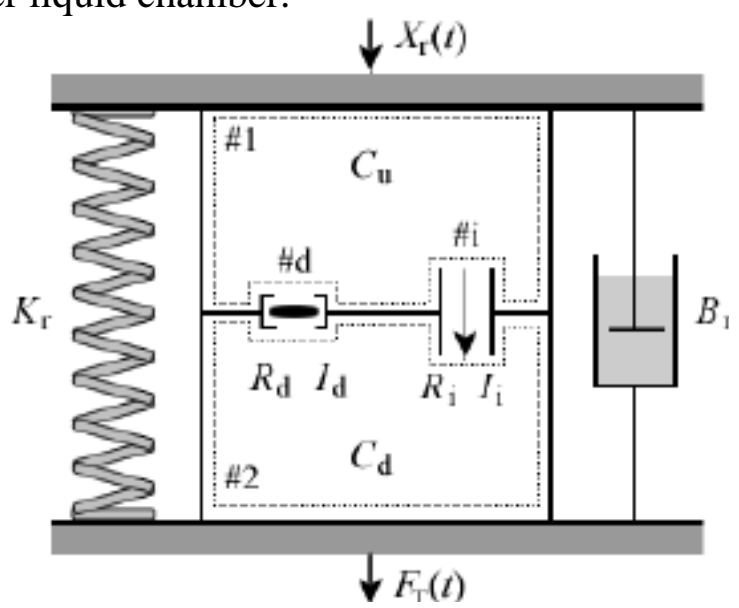

Fig. 1 Mechanical model of HEM with decoupler 
The continuity equation flowed in the upper and lower chamber was established according to figure 1, as following:

$C_{u} \dot{P}_{1}=A_{p} \dot{X}_{t}-Q_{i}-Q_{d}$

$C_{d} \dot{P}_{2}=Q_{i}+Q_{d}$

The momentum equation of the inertia channel is

$$
P_{1}-P_{2}=I_{i} \dot{Q}_{1}+R_{i} Q_{i}
$$

The momentum equation of the decoupling channel is

$$
P_{1}-P_{2}=I_{d} \dot{Q}_{d}+R_{d} Q_{d}
$$

The vibration force of the body from the powertrain transmitted through hydraulic suspension is:

$$
F_{T}=B_{r} \dot{X}_{r}+K_{r} X_{r}+A_{p} P_{1}
$$

Taking $u=\dot{X}_{r}$ as the input variable, $y=F_{T}$ as the output variable, and $x=\left[\begin{array}{lllll}X_{r} & P_{1} & P_{2} & Q_{i} & Q_{d}\end{array}\right]^{T}$ as the state variable, the state equation of the system can be established as following:

$\left\{\begin{array}{l}\dot{x}(t)=A x(t)+B u(t) \\ y(t)=C x(t)+D u(t)\end{array}\right.$

Where,

$A=\left[\begin{array}{ccccc}0 & 0 & 0 & 0 & 0 \\ 0 & 0 & 0 & -1 / C_{u} & -1 / C_{u} \\ 0 & 0 & 0 & 1 / C_{d} & 1 / C_{d} \\ 0 & 1 / I_{i} & -1 / I_{i} & -R_{i} / I_{i} & 0 \\ 0 & 1 / I_{d} & -1 / I_{d} & 0 & -R_{d} / I_{d}\end{array}\right] \quad B=\left[\begin{array}{c}1 \\ A_{p} / C_{u} \\ 0 \\ 0 \\ 0\end{array}\right]$

$C=\left[\begin{array}{lllll}K_{r} & A_{p} & 0 & 0 & 0\end{array}\right] \quad D=\left[B_{r}\right]$

The equation (6) was transformed by the Laplace transform, as following:

$\left\{\begin{array}{l}s I X(s)=A X(s)+B U(s) \\ Y(s)=C X(s)+D U(s)\end{array}\right.$

Where, $I$ is the unit matrix which is the same as the order of the matrix A.

The equation (7) yields the formula:

$$
X(s)=(s I-A)^{-1} B U(s)
$$

The equation (7) and (8) yields the system transfer function as following

$G(s)=Y / U=C(s I-A)^{-1} B+D$

Then the hydraulic mount complex stiffness is:
$K(s)=\frac{F_{T}(s)}{X_{r}(s)}=\frac{Y s}{U}=(s I-A)^{-1} B s+D s$

Put $s=j \omega$, into equation (10) is:

$K(j \omega)=C(j \omega \cdot I-A)^{-1} B \cdot j \omega+D \cdot j \omega$

The formula (11) settled can be expressed as

$K(j \omega)=k^{\prime}(\omega)+j \cdot k^{\prime \prime}(\omega)$

Where, $k^{\prime}(\omega)$ and $k^{\prime \prime}(\omega)$ are respectively the storage stiffness and the loss of stiffness of the hydraulic suspension.

The dynamic stiffness $k_{d}$ and the lag angle $\varphi$ of the hydraulic mount can be expressed as

$k_{d}=\sqrt{k^{\prime}(\omega)+k^{\prime \prime}(\omega)}$

$\varphi=\arctan \left(k^{\prime \prime}(\omega) / k^{\prime}(\omega)\right)$

The dynamic stiffness reflects the amplitude ratio under the excitation frequency between the response force and the displacement excitation[3]. The lag angle reflects the hysteresis angle that the displacement lags the force under the excitation frequency, which is used to characterize the damping of the hydraulic suspension. The common damping coefficient $c_{d}$ used in engineering can be expressed as:

$$
c_{d}=k_{d} \sin \varphi / \omega
$$

\section{SIMULATION MODEL OF THE HYDRAULIC MOUNT}

According to the switched system model of the decoupling disc hydraulic suspension, the simulation model using Simulink module in Matlab software was established such as figure 2, and the mode switched simulation realized by Stateflow[4].

\subsection{Dynamic characteristics simulation model}

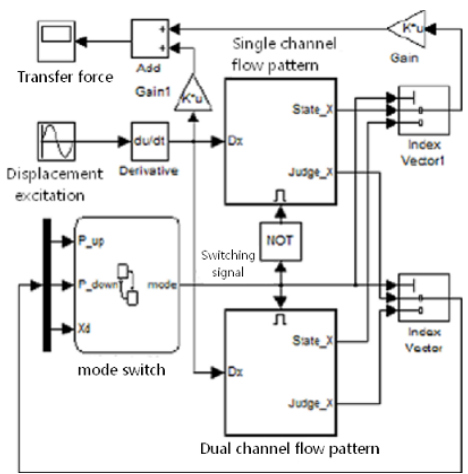

Fig. 2 Dynamic characteristics simulation model based on switched system 
Table 1 Simulation parameters

\begin{tabular}{|c|c|c|c|c|c|c|c|c|c|}
\hline $\mathrm{Kr}$ & $1.188 \times 105 \mathrm{~N} / \mathrm{m}$ & $\mathrm{Cu}$ & $2.234 \times 10-11 \mathrm{~m} 5 / \mathrm{N}$ & $\mathrm{Ri}$ & $2.924 \times 108 \mathrm{~N} \cdot \mathrm{s} / \mathrm{m} 5$ & $\mathrm{Am}$ & $7.065 \times 10-4 \mathrm{~m} 2$ & $\mathrm{~h}$ & $2.7 \mathrm{~mm}$ \\
\hline $\mathrm{Br}$ & $890 \mathrm{~N} \cdot \mathrm{s} / \mathrm{m}$ & $\mathrm{Cd}$ & $2.825 \times 10-9 \mathrm{~m} 5 / \mathrm{N}$ & $\mathrm{Id}$ & $1.721 \times 105 \mathrm{~kg} / \mathrm{m} 4$ & $\mathrm{md}$ & $0.01 \mathrm{~kg}$ & & \\
\hline $\mathrm{Ap}$ & $1.521 \times 10-3 \mathrm{~m} 2$ & $\mathrm{Ii}$ & $4.196 \times 106 \mathrm{~kg} / \mathrm{m} 4$ & $\mathrm{Rd}$ & $6.242 \times 107 \mathrm{~N} \cdot \mathrm{s} / \mathrm{m} 5$ & $\mathrm{kd}$ & $1.77 \times 104 \mathrm{~N} / \mathrm{m}$ & & \\
\hline
\end{tabular}

The simulation parameters, from the above analysis, required by the model have the main rubber spring stiffness $K_{r}$, the damping coefficient $B_{r}$ and its equivalent piston area $A_{p}$, the up and down liquid chamber volume compliance of $C_{u}$ and $C_{d}$, the inertia $I_{i}$ and the damping coefficient $R_{i}$ of the liquid in the inertia channel, the inertial coefficient $I_{d}$ and the damping coefficient $R_{d}$ of the liquid in the decoupling channel, the decoupling disc area $A_{m}$, the decoupling disc and its additional liquid mass $m_{d}$ and the stiffness $k_{d}$, and the half width $h$ of the clearance in decoupling channel. Where $A_{m}, m_{d}$ and $h$ can be measured directly, the rest parameters be obtained by using the finite element method, the parameter values as shown in table 1 .

\section{VERIFICATION OF SIMULATION PRE- EXPERIMENTAL}

The dynamic characteristics simulation of the decoupling disc hydraulic mount was tested in two kinds of typical condition respectively, and compared with the test results, as shown in Figure 3 and 4.

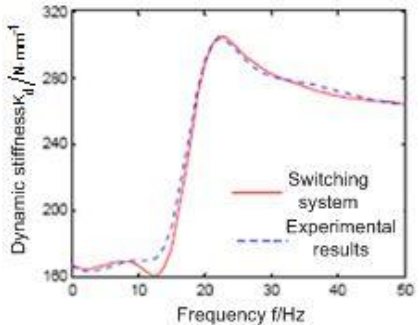

(a) Dynamic stiffness

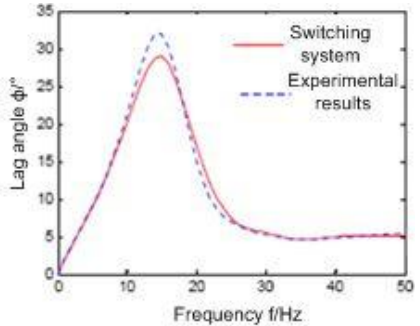

(b) Lag angle
Fig. 3 Low-frequency performance comparison between calculation and test results

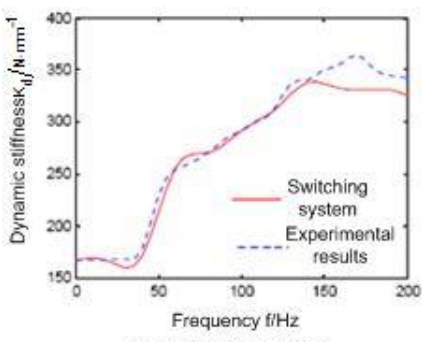

(a) Dynamic stiffness

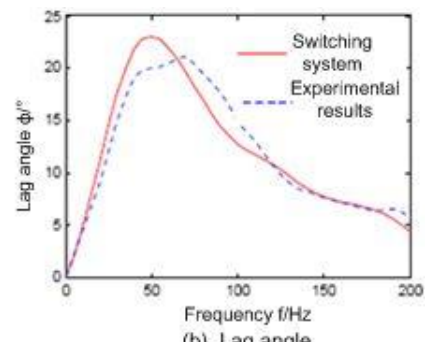

(b) Lag angle
Fig. 4 High-frequency performance comparison between calculation and test results
Figure 3 shows that the dynamic characteristics simulation results of the switched system model in the low-frequency extremely closed the experimental results on the whole and the mean relative error of the dynamic stiffness and the lag angle were respectively $1.43 \%$ and $4.99 \%$. The dynamic characteristics of curve did not fit ideally when the frequency range was in $10 \mathrm{~Hz} \sim 20 \mathrm{~Hz}$. Under the low frequency and the large amplitude conditions, the lower the excitation frequency, the more time the hydraulic mounts worked in the dual channel mode which leaded to the more liquid would flow the decoupling channel. The decoupling channel was considered as the concentric annular aperture in modeling, however the practical work process was the eccentric annular aperture and unstable. So the more liquid flowed through the decoupling channel the more difference between the simulation results and the tested results. When the frequency range was in $10 \mathrm{~Hz} \sim 20 \mathrm{~Hz}$, the liquid flowed slowly between the upper and the lower liquid chamber which reduced the effect of the liquid damping, the dynamic characteristics of the hydraulic mount mainly reflects the dynamic characteristic of the main rubber spring, so the calculation results of the switched system model fit basically the test results within this band [5].

The figure 4 shows that the dynamic characteristics simulation results of the switched system model in the high-frequency well closed the experimental results on the whole and the mean relative error of the dynamic stiffness and the lag angle were respectively $3.03 \%$ and $9.97 \%$. But there is a certain deviation when the dynamic stiffness curves above $145 \mathrm{~Hz}$. The measured dynamic stiffness curve peaked near $170 \mathrm{~Hz}$, showing the subsystem resonance phenomenon occurred in the liquid damping mechanism, and the switched system model also showed this, but the peak frequency was at $145 \mathrm{~Hz}$, slightly smaller than actually measured value. With the increase of the frequency, the flow speed through the decoupling channel increased. The critical Reynolds number of the liquid exceeded the critical Reynolds number in the decoupling channel that made the liquid of the up liquid chamber became very complex and uneven distribution of the stress, which had obvious influence on the dynamic characteristics of the hydraulic mount. 


\section{CONCLUSIONS}

The decoupling disc hydraulic mount switched system model was established. The dynamic characteristic test of the homemade decoupling disc hydraulic mount were performed, obtaining the dynamic characteristics under different working conditions and providing theoretical reference to reveal the working mechanism of hydraulic mount and the structure design.

Finally according to the decoupling disc simulation in two kinds of typical working condition and comparing with the test result validated the effectiveness of the switched system model.

\section{ACNOWLEDGEMENT}

The authors would like to thank the financial supports of Anhui Provincial Natural Science Foundation (Grant No. 1508085 ME70) and teacher studio, 2014 colleges and universities in Anhui province quality project,(Grant No. 2014 msgzs189), project leader: Yaqin Wang.

\section{REFERENCES}

[1] J. F. Hu, R. Singh. Improved Troque Roll Axis Decoupling Axiom for A Powertrain Mounting System in the Presence of A Compliant Base. Journal of Sound and Vibration, 2012, 331(7):1498-1518.

[2] Shi Wenku, Mao Yang, Jiang Xue, Chen Zhiyong, Ma Hongli, Pan Bin. Study on Dynamic Charactrtistics and Parameter Influence of Engine's Semi-Active Hydraulic Mount Using AMESim. Journal of Xi'an Jiao Tong University, 2014, 48(1):42-47.(In Chinese)

[3] Shi Peicheng, Chen Wuwei, Jiang Wuhua. Simulation Research on Hydraulic Mount Nonlinear Dynamic Characteristics. Chinese Mechanical Engineering, 2009, 24(2):2545-2548. (In Chinese)

[4] Li Qian. Study on the Dynamic Characteristic and Parameter Identification of Hydraulic Engine Mount in Automotive Powertrain Mounting System. Shanghai: Shanghai Jiao Tong University, 2010. (In Chinese)

[5] Shu Changdong. Study on the Dynamic Characteristics and A Simulation of Hydraulic Engine Mount in Automotive Engine Mounting System. Shanghai: Donghua University, 2012. (In Chinese) 